\title{
Polynomial Automorphisms and the Jacobian Conjecture
}

\author{
Arno van den ESSEN*
}

\begin{abstract}
In this paper we give an update survey of the most important results concerning the Jacobian conjecture: several equivalent descriptions are given and various related conjectures are discussed. At the end of the paper, we discuss the recent counter-examples, in all dimensions greater than two, to the Markus-Yamabe conjecture (Global asymptotic Jacobian conjecture).
\end{abstract}

\section{Résumé}

Dans ce papier nous présentons un rapport actualisé sur les résultats les plus importants concernant la conjecture Jacobienne : plusieurs formulations équivalentes et diverses conjectures connexes sont considérées. A la fin du papier, nous donnons les contre-exemples récents, en toute dimension plus grande que deux, à la conjecture de Markus-Yamabe.

\section{Introduction}

The last fifteen years the interest in the study of polynomial automorphisms is growing rapidly. The main motivation behind this interest is the existence of several very appealing open problems such as the tame generators conjecture, some linearization problems and last but not least the Jacobian Conjecture.

The aim of this paper is to give a survey of the Jacobian Conjecture, including the most recent results (up to date).

The paper is divided into three parts. In the first chapter a short survey is given of the most important $n$-dimensional results concerning the Jacobian Conjecture. In the second chapter we study the Jacobian Conjecture from the viewpoint of derivations and relate it to a conjecture about the kernel of a derivation. It turns out that the cases of dimension two and that of dimension bigger than two are essentially different. Finally in the third chapter we discuss some important problems and indicate how they are related to the Jacobian Conjecture.

\footnotetext{
AMS 1980 Mathematics Subject Classification (1985 Revision): 14E09

${ }^{*}$ Department of Mathematics, University of Nijmegen, 6525 ED Nijmegen, The Netherlands
} 


\section{Acknowledgement}

I like to thank the organizers for the possibility of participating in this perfectly organised conference!

\section{The Jacobian Conjecture: a short survey}

From calculus everyone knows the classical Rolle theorem:

Theorem 1.1 - If $F: \mathbb{R} \rightarrow \mathbb{R}$ is a $\mathscr{C}^{1}$-function such that $F(a)=F(b)$ for some $a \neq b$ in $\mathbb{R}$, then there exists a point $z \in \mathbb{R}$ such that $F^{\prime}(z)=0$.

The main question of this paper concerns an attempt to generalise this result in a certain direction. More precisely, let $F=\left(F_{1}, \ldots, F_{n}\right): \mathbb{C}^{n} \rightarrow \mathbb{C}^{n}$ be a polynomial map, i.e. a map of the form

$$
\left(x_{1}, \ldots, x_{n}\right) \mapsto\left(F_{1}\left(x_{1}, \ldots, x_{n}\right), \ldots, F_{n}\left(x_{1}, \ldots, x_{n}\right)\right)
$$

where each $F_{i} \in \mathbb{C}[X]:=\mathbb{C}\left[X_{1}, \ldots, X_{n}\right]$, the $n$ variable polynomial ring over $\mathbb{C}$. Furthermore for $z \in \mathbb{C}^{n}$ put $F^{\prime}(z):=\operatorname{det}(J F(z))$ where

$$
J F=\left(\frac{\partial F_{i}}{\partial X_{j}}\right)_{1 \leq i, j \leq n}
$$

is the Jacobian matrix over $F$. Now the main question is:

Question 1.2 - Let $F(a)=F(b)$ for some $a, b \in \mathbb{C}^{n}$ with $a \neq b$. Does it follow that $F^{\prime}(z)=0$ for some $z \in \mathbb{C}^{n}$.

The answer (at this moment) is: we don't know if $n \geq 2$ ! In fact this question is, as we will show below, a reformulation of the famous Jacobian Conjecture.

Conjecture 1.3 (Jacobian Conjecture $(J C(n))$ ) - Let $F: \mathbb{C}^{n} \rightarrow \mathbb{C}^{n}$ be a polynomial map such that $F^{\prime}(z) \neq 0$ for all $z \in \mathbb{C}^{n}$ (or equivalently $\operatorname{det}(J F) \in \mathbb{C}^{*}$ ), then $F$ is invertible (i.e. $F$ has an inverse which is also a polynomial map).

To see that the above Rolle type question is indeed equivalent to the Jacobian Conjecture, we recall the following beautiful result due to Białynicki-Birula and Rosenlicht [7], 1962.

Theorem 1.4 (Biatynicki-Birula, Rosenlicht) - Let $k$ be an algebraically closed field of characteristic zero. Let $F: k^{n} \rightarrow k^{n}$ be a polynomial map. If $F$ is injective, then $F$ is surjective and the inverse is a polynomial map, i.e. $F$ is a polynomial automorphism.

So the Jacobian Conjecture is equivalent to: if $F^{\prime}(z) \neq 0$ for all $z \in \mathbb{C}^{n}$, then $F$ is injective or equivalently if $F(a)=F(b)$ for some $a \neq b, a, b \in \mathbb{C}^{n}$ then $F^{\prime}(z)=0$ for 
some $z \in \mathbb{C}^{n}$, which is exactly the 'Rolle form' of the Jacobian Conjecture described in the question above.

The Jacobian Conjecture was first formulated as a question by O. Keller in the case $n=2$ for polynomials with integer coefficients ([35], 1939). Therefore the Jacobian Conjecture is also called Keller's problem by several authors. Over the years many people have tried to prove the Jacobian Conjecture. As a result many false proofs have been given and even several of them are published (for an account on these 'proofs' we refer to the paper [4]). But more importantly the study of the Jacobian Conjecture has given rise to several surprising results concerning polynomial automorphisms and many interesting relations with other problems.

In the remainder of this section we will describe the present status of the $n$ dimensional Jacobian Conjecture.

So from now on let $F=\left(F_{1}, \ldots, F_{n}\right): \mathbb{C}^{n} \rightarrow \mathbb{C}^{n}$ be a polynomial map. Put

$$
\operatorname{deg}(F):=\max _{i} \operatorname{deg}\left(F_{i}\right)
$$

where $\operatorname{deg}\left(F_{i}\right)$ means the total degree of $F_{i}$.

From linear algebra we know that the Jacobian Conjecture is true if $\operatorname{deg}(F)=1$. So the next case is $\operatorname{deg}(F)=2$. It was only in 1980 that Stuart Wang proved that in this case the Jacobian Conjecture is true:

Proposition 1.5 (Wang, [58]) - If $\operatorname{deg}(F) \leq 2$, then the Jacobian Conjecture is true.

Proof. By theorem 1.4 it suffices to prove that $F$ is injective. So suppose $F(a)=F(b)$ for some $a, b \in \mathbb{C}^{n}, a \neq b$. We first show that we can assume that $b=0$. To see this we define $G(X):=F(X+a)-F(a)$. Then $\operatorname{deg}(G) \leq 2, G(0)=0$ and putting $c:=b-a$ we have $c \neq 0$ and $G(c)=0$. Observe $J G(X)=(J F)(X+a)$, so $\operatorname{det}(J G) \in \mathbb{C}^{*}$. Now write $G=G_{(1)}+G_{(2)}$, its decomposition in homogeneous components. Consider $G(t c)=t G_{(1)}(c)+t^{2} G_{(2)}(c)$. Differentiation gives

$$
G_{(1)}(c)+2 t G_{(2)}(c)=\frac{d}{d t} G(t c)=J G(t c) \cdot c \neq 0
$$

for all $t \in \mathbb{C}$, since $c \neq 0$ and $\operatorname{det}(J G) \in \mathbb{C}^{*}$. Substituting $t=\frac{1}{2}$ gives $G(c) \neq 0$, a contradiction with $G(c)=0$. So $F$ is injective.

Now one could think that this result is just a small improvement of the case $\operatorname{deg}(F)=1$. However we have

Theorem 1.6 (Bass, Connell, Wright, [4], Yagzhev, [61]) - If the Jacobian Conjecture holds for all $n \geq 2$ and all $F$ with $\operatorname{deg}(F) \leq 3$, then the Jacobian Conjecture holds. 
In fact they even proved that it suffices to prove the Jacobian Conjecture for all $n \geq 2$ and all $F$ of the form

$$
F=\left(X_{1}+H_{1}, \ldots, X_{n}+H_{n}\right)
$$

where each $H_{i}$ is either zero or homogeneous of degree 3.

A little later this result was improved by Drużkowski:

Theorem 1.7 (Drużkowski, [17]) - If the Jacobian Conjecture holds for all $n \geq 2$ and all $F$ of the form

$$
F=\left(X_{1}+\left(\sum a_{j 1} X_{j}\right)^{3}, \ldots, X_{n}+\left(\sum a_{j n} X_{j}\right)^{3}\right)
$$

then the Jacobian Conjecture holds.

What is known about the Jacobian Conjecture for the maps of the form (1) resp. (2)?

In 1993 David Wright in [60] showed that in case $n=3$ the Jacobian Conjecture holds for all $F$ of the form (1). In that paper Wright writes:

'Here it becomes useful to assume $F$ is cubic homogeneous, since this limits the number of its monomials. The dimension four case may still be out of range even with this reduction, however; the number of monomials of degree three in four variables is 20 , so the number of monomials for a cubic homogeneous map in dimension four is $20 \times 4=80$.'

Nevertheless Engelbert Hubbers (University of Nijmegen) succeeded in 1994 to solve the large system of polynomial equations (induced by $\operatorname{det}(J F)=1$ ) with the help of a strong computer. So he showed that in case $n=4$ the Jacobian Conjecture holds for all $F$ of the form (1). In fact he completely classified all maps of the form (1) satisfying $\operatorname{det}(J F)=1$. His main result is

Theorem 1.8 (Hubbers, [31]) - Let $F=X-H$ be a cubic homogeneous polynomial map in dimension four, such that $\operatorname{det}(J F)=1$. Then there exists some $T \in G L_{4}(\mathbb{C})$ with $T^{-1} \circ F \circ T$ being one of the following forms:

$$
\text { 1. }\left(\begin{array}{cc}
x_{1} & \\
x_{2} & \\
x_{3} & \\
x_{4} & -a_{4} x_{1}^{3}-b_{4} x_{1}^{2} x_{2}-c_{4} x_{1}^{2} x_{3}-e_{4} x_{1} x_{2}^{2}-f_{4} x_{1} x_{2} x_{3} \\
& -h_{4} x_{1} x_{3}^{2}-k_{4} x_{2}^{3}-l_{4} x_{2}^{2} x_{3}-n_{4} x_{2} x_{3}^{2}-q_{4} x_{3}^{3}
\end{array}\right)
$$


2. $\left(\begin{array}{cc}x_{1} & \\ x_{2} & -\frac{1}{3} x_{1}^{3}-h_{2} x_{1} x_{3}^{2}-q_{2} x_{3}^{3} \\ x_{3} & \\ x_{4} & -x_{1}^{2} x_{3}-h_{4} x_{1} x_{3}^{2}-q_{4} x_{3}^{3}\end{array}\right)$

$3 .\left(\begin{array}{ll}x_{1} & \\ x_{2} & -\frac{1}{3} x_{1}^{3}-c_{1} x_{1}^{2} x_{4}+3 c_{1} x_{1} x_{2} x_{3}-\frac{16 q_{4} c_{1}^{2}-r_{4}^{2}}{48 c_{1}^{2}} x_{1} x_{3}^{2}-\frac{1}{2} r_{4} x_{1} x_{3} x_{4} \\ & +\frac{3}{4} r_{4} x_{2} x_{3}^{2}-\frac{r_{4} q_{4}}{12 c_{1}} x_{3}^{3}-\frac{r_{4}^{2}}{16 c_{1}} x_{3}^{2} x_{4} \\ x_{3} & \\ x_{4} & -x_{1}^{2} x_{3}+\frac{r_{4}}{4 c_{1}} x_{1} x_{3}^{2}-3 c_{1} x_{1} x_{3} x_{4}+9 c_{1} x_{2} x_{3}^{2}-q_{4} x_{3}^{3}-\frac{3}{4} r_{4} x_{3}^{2} x_{4}\end{array}\right)$

4. $\left(\begin{array}{ll}x_{1} & \\ x_{2} & -\frac{1}{3} x_{1}^{3} \\ x_{3} & -x_{1}^{2} x_{2}-e_{3} x_{1} x_{2}^{2}-k_{3} x_{2}^{3} \\ x_{4} & -e_{4} x_{1} x_{2}^{2}-k_{4} x_{2}^{3}\end{array}\right)$

$5 .\left(\begin{array}{ll}x_{1} & \\ x_{2} & -\frac{1}{3} x_{1}^{3}+i_{3} x_{1} x_{2} x_{4}-j_{2} x_{1} x_{4}^{2}+s_{3} x_{2} x_{4}^{2}+i_{3}^{2} x_{3} x_{4}^{2}-t_{2} x_{4}^{3} \\ x_{3} & -x_{1}^{2} x_{2}-\frac{2 s_{3}}{i_{3}} x_{1} x_{2} x_{4}-i_{3} x_{1} x_{3} x_{4}-j_{3} x_{1} x_{4}^{2}-\frac{s_{3}^{2}}{i_{3}^{2}} x_{2} x_{4}^{2} \\ & -s_{3} x_{3} x_{4}^{2}-t_{3} x_{4}^{3} \\ x_{4} & \end{array}\right)$

6. $\left(\begin{array}{ll}x_{1} & \\ x_{2} & -\frac{1}{3} x_{1}^{3}-j_{2} x_{1} x_{4}^{2}-t_{2} x_{4}^{3} \\ x_{3} & -x_{1}^{2} x_{2}-e_{3} x_{1} x_{2}^{2}-g_{3} x_{1} x_{2} x_{4}-j_{3} x_{1} x_{4}^{2}-k_{3} x_{2}^{3}-m_{3} x_{2}^{2} x_{4} \\ & -p_{3} x_{2} x_{4}^{2}-t_{3} x_{4}^{3} \\ x_{4} & \end{array}\right)$

7. $\left(\begin{array}{ll}x_{1} & \\ x_{2} & -\frac{1}{3} x_{1}^{3} \\ x_{3} & -x_{1}^{2} x_{2}-e_{3} x_{1} x_{2}^{2}-k_{3} x_{2}^{3} \\ x_{4} & -x_{1}^{2} x_{3}-e_{4} x_{1} x_{2}^{2}-f_{4} x_{1} x_{2} x_{3}-h_{4} x_{1} x_{3}^{2}-k_{4} x_{2}^{3}-l_{4} x_{2}^{2} x_{3} \\ & -n_{4} x_{2} x_{3}^{2}-q_{4} x_{3}^{3}\end{array}\right)$ 
$8 .\left(\begin{array}{ll}x_{1} & \\ x_{2} & -\frac{1}{3} x_{1}^{3} \\ x_{3} & -x_{1}^{2} x_{2}-e_{3} x_{1} x_{2}^{2}+g_{4} x_{1} x_{2} x_{3}-k_{3} x_{2}^{3}+m_{4} x_{2}^{2} x_{3}+g_{4}^{2} x_{2}^{2} x_{4} \\ x_{4} & -x_{1}^{2} x_{3}-e_{4} x_{1} x_{2}^{2}-\frac{2 m_{4}}{g_{4}} x_{1} x_{2} x_{3}-g_{4} x_{1} x_{2} x_{4}-k_{4} x_{2}^{3} \\ & -\frac{m_{4}^{2}}{g_{4}^{2}} x_{2}^{2} x_{3}-m_{4} x_{2}^{2} x_{4}\end{array}\right)$

Combining this result with earlier results of Drużkowski in [18], he deduced that Corollary 1.9 (Hubbers, [31]) - The Jacobian Conjecture holds for all $F$ of the form (2) if $n \leq 7$.

Since the paper [31] is not easy accessible the proof of this result is reprinted in [20] (cf. Proposition 2.9 and the corollaries 2.11 and 2.12).

Another remarkable result was obtained by Jie-Tai Yu ([62], 1995). To describe his result we need some preparations. Let $F=\left(F_{1}, \ldots, F_{n}\right): \mathbb{C}^{n} \rightarrow \mathbb{C}^{n}$ be a polynomial map. Then consider the map $\tilde{F}: \mathbb{R}^{2 n} \rightarrow \mathbb{R}^{2 n}$ defined by $\tilde{F}=$ $\left(\operatorname{Re} F_{1}, \operatorname{Im} F_{1}, \ldots, \operatorname{Re} F_{n}, \operatorname{Im} F_{n}\right)$. It is well-known that $\operatorname{det}(J \tilde{F})=|\operatorname{det}(J F)|^{2}$. So $\operatorname{det}(J F) \in \mathbb{C}^{*}$ if and only if $\operatorname{det}(J \tilde{F}) \in \mathbb{R}^{*}$. Obviously $F$ is injective if and only if $\tilde{F}$ is injective. Consequently if the Jacobian Conjecture holds for all (real coefficients) polynomial maps from $\mathbb{R}^{n} \rightarrow \mathbb{R}^{n}$, for all $n \geq 2$, then the Jacobian Conjecture holds (use theorem 1.4). So it is no restriction to study only polynomial maps from $\mathbb{R}^{n} \rightarrow \mathbb{R}^{n}$ such that $\operatorname{det}(J F) \in \mathbb{R}^{*}$. Of course we may also assume that $F(0)=0$ and $J F(0)=I$. So we can write

$$
F=X+F_{(2)}+\cdots+F_{(d)}
$$

its homogeneous decomposition.

Definition 1.10 - A polynomial map of the form (3) is called positive (resp. negative) if all non-zero coefficients of the $F_{(i)}$ are postitive (resp. negative).

Now we have the following two results.

Theorem 1.11 (Yu, [62]) - If for all $n \geq 2$ and all positive $F: \mathbb{R}^{n} \rightarrow \mathbb{R}^{n}$ with $\operatorname{det}(J F)=1, F$ is injective, then the Jacobian Conjecture holds.

$\mathrm{Yu}$ could not prove the injectivity for positive $F$ 's, however he obtained the following result:

Theorem 1.12 (Yu, [62]) - For all $n \geq 2$ and all negative $F: \mathbb{R}^{n} \rightarrow \mathbb{R}^{n}$ with $\operatorname{det}(J F)=1$ the Jacobian Conjecture is true. Even stronger, each such $F$ is stably tame (i.e. for suitable $m \in \mathbb{N}$ the extended map

$$
F^{[m]}=\left(F_{1}, \ldots, F_{n}, Y_{1}, \ldots, Y_{m}\right)
$$

is a product of elementary polynomial maps). 
Finally we like to mention the following $n$-dimensional result

Theorem 1.13 (Lang, Maslamani, [39]) - Let $k$ be a field with $\operatorname{char}(k)=0$ and let $F_{1}, \ldots, F_{n} \in k[X]$ with $\operatorname{det}(J F) \in k^{*}$.

1. If $F_{i} \in X_{i} k[X]$ for all $i$, then $F_{i}=\lambda_{i} X_{i}$ with $\lambda_{i} \in k^{*}$, so $F$ is invertible.

2. If $F_{i}=X_{i}+\lambda_{i} M_{i}$ for all $i$, where $\lambda_{i} \in k$ and $M_{i}$ is a monomial, then $F$ is invertible.

\section{Derivations and the Jacobian Conjecture}

\subsection{Derivations and the Jacobian Condition}

The aim of this section is to study the Jacobian Conjecture by means of derivations. Therefore we first reformulate the Jacobian Conjecture in terms of the kernel of a special derivation.

Let $F=\left(F_{1}, \ldots, F_{n}\right): \mathbb{C}^{n} \rightarrow \mathbb{C}^{n}$ be a polynomial map satisfying the Jacobian condition, i.e. $\operatorname{det}(J F) \in \mathbb{C}^{*}$. To such a map we associate an $n$-tuple of derivations on $\mathbb{C}[X]$, denoted by $\frac{\partial}{\partial F_{1}}, \ldots, \frac{\partial}{\partial F_{n}}$ as follows

$$
\left(\begin{array}{c}
\frac{\partial}{\partial F_{1}} \\
\vdots \\
\frac{\partial}{\partial F_{n}}
\end{array}\right)=\left((J F)^{-1}\right)^{T}\left(\begin{array}{c}
\frac{\partial}{\partial X_{1}} \\
\vdots \\
\frac{\partial}{\partial x_{n}}
\end{array}\right)
$$

One readily verifies from the definitions that

$$
\frac{\partial}{\partial F_{i}}\left(F_{j}\right)=\delta_{i j}
$$

for all $i, j$.

Lemma 2.1 (Nousiainen, Sweedler, [51]) - The derivations $\frac{\partial}{\partial F_{1}}, \ldots, \frac{\partial}{\partial F_{n}}$ form a $\mathbb{C}[X]$-basis of $\operatorname{Der}_{\mathbb{C}} \mathbb{C}[X]$ which is commutative i.e. $\left[\frac{\partial}{\partial F_{i}}, \frac{\partial}{\partial F_{j}}\right]=0$ for all $i, j$.

Proof. $\quad$ 1. Since $\frac{\partial}{\partial x_{1}}, \ldots, \frac{\partial}{\partial x_{n}}$ is a $\mathbb{C}[X]$-basis of $\operatorname{Der}_{\mathbb{C}} \mathbb{C}[X]$ and $J F$ is invertible, the first statement immediately follows from (4).

2. Choose $i, j$. Put $d:=\left[\frac{\partial}{\partial F_{i}}, \frac{\partial}{\partial F_{j}}\right]$. By (5) it follows that $d\left(F_{i}\right)=0$ for all $i$. By 1., $d$ can be written as $d=\sum c_{i} \frac{\partial}{\partial F_{i}}$ for some $c_{i} \in \mathbb{C}[X]$. Since by (5) $c_{i}=d\left(F_{i}\right)$ we deduce that $c_{i}=0$ for all $i$, whence $d=0$.

In fact the two properties described in lemma 2.1 completely characterize the derivations $\frac{\partial}{\partial F_{1}}, \ldots, \frac{\partial}{\partial F_{n}}$ associated to a polynomial map satisfying the Jacobian condition. More precisely 
Proposition 2.2 (Nowicki, [52]) - Let $D_{1}, \ldots, D_{n}$ be a commutative $\mathbb{C}[X]$-basis of $\operatorname{Der}_{\mathbb{C}} \mathbb{C}[X]$. Then there exists a polynomial map $F=\left(F_{1}, \ldots, F_{n}\right)$ with $\operatorname{det}(J F) \in$ $\mathbb{C}^{*}$ such that $D_{i}=\frac{\partial}{\partial F_{i}}$ for all $i$.

Proof. Since $D_{1}, \ldots, D_{n}$ is a $\mathbb{C}[X]$-basis of $\operatorname{Der}_{\mathbb{C}} \mathbb{C}[X]$ we get

$$
\left(\begin{array}{c}
\partial_{1} \\
\vdots \\
\partial_{n}
\end{array}\right)=B\left(\begin{array}{c}
D_{1} \\
\vdots \\
D_{n}
\end{array}\right)
$$

where

$$
B=\left(b_{i j}\right) \in \mathrm{GL}_{n}(\mathbb{C}[X])
$$

i.e. $\operatorname{det}(B) \in \mathbb{C}^{*}$. Now write the equations $\left[\partial_{i}, \partial_{j}\right]$ in terms of the $b_{i j}$ and the derivations $D_{j}$ and use the hypothesis that $\left[D_{i}, D_{j}\right]=0$ for all $i, j$. This gives

$$
\begin{aligned}
& \left(b_{i 1} D_{1}\left(b_{j 1}\right)+\cdots+b_{i n} D_{n}\left(b_{j 1}\right)\right) D_{1}+\cdots+\left(b_{i 1} D_{1}\left(b_{j n}\right)+\cdots+b_{i n} D_{n}\left(b_{j n}\right)\right) D_{n} \\
= & \left(b_{j 1} D_{1}\left(b_{i 1}\right)+\cdots+b_{j n} D_{n}\left(b_{i 1}\right)\right) D_{1}+\cdots+\left(b_{j 1} D_{1}\left(b_{i n}\right)+\cdots+b_{j n} D_{n}\left(b_{i n}\right)\right) D_{n}
\end{aligned}
$$

Equating the corresponding coefficients of the $D_{i}$ (using that $D_{1}, \ldots, D_{n}$ forms a $\mathbb{C}[X]$-basis of $\left.\operatorname{Der}_{\mathbb{C}} \mathbb{C}[X]\right)$ we get

$$
\begin{array}{ccc}
b_{i 1} D_{1}\left(b_{j 1}\right)+\cdots+b_{i n} D_{n}\left(b_{j 1}\right) & = & b_{j 1} D_{1}\left(b_{i 1}\right)+\cdots+b_{j n} D_{n}\left(b_{i 1}\right) \\
\vdots & \vdots & \vdots \\
b_{i 1} D_{1}\left(b_{j n}\right)+\cdots+b_{i n} D_{n}\left(b_{j n}\right) & = & b_{j 1} D_{1}\left(b_{i n}\right)+\cdots+b_{j n} D_{n}\left(b_{i n}\right)
\end{array}
$$

or equivalently

$$
\left(b_{i 1} D_{1}+\cdots+b_{i n} D_{n}\right)\left(\begin{array}{c}
b_{j 1} \\
\vdots \\
b_{j n}
\end{array}\right)=\left(b_{j 1} D_{1}+\cdots+b_{j n} D_{n}\right)\left(\begin{array}{c}
b_{i 1} \\
\vdots \\
b_{i n}
\end{array}\right)
$$

i.e.

$$
\partial_{i}\left(\begin{array}{c}
b_{j 1} \\
\vdots \\
b_{j n}
\end{array}\right)=\partial_{j}\left(\begin{array}{c}
b_{i 1} \\
\vdots \\
b_{i n}
\end{array}\right)
$$

for all $i, j$. So by Poincarré's lemma

$$
B^{T}=\left(\partial_{1}\left(\begin{array}{c}
F_{1} \\
\vdots \\
F_{n}
\end{array}\right), \ldots, \partial_{n}\left(\begin{array}{c}
F_{1} \\
\vdots \\
F_{n}
\end{array}\right)\right)
$$


for some $F_{1}, \ldots, F_{n}$ in $\mathbb{C}[X]$. So $B^{T}=J F$ and hence by (6) and (7) we get

$$
\left(\begin{array}{c}
D_{1} \\
\vdots \\
D_{n}
\end{array}\right)=\left((J F)^{T}\right)^{-1}\left(\begin{array}{c}
\partial_{1} \\
\vdots \\
\partial_{n}
\end{array}\right)
$$

with $\operatorname{det}(J F) \in \mathbb{C}^{*}$.

Corollary 2.3 - The Jacobian Conjecture is equivalent to saying that apart from a polynomial coordinate change $\left(\frac{\partial}{\partial X_{1}}, \ldots, \frac{\partial}{\partial X_{n}}\right)$ is the only commutative $\mathbb{C}[X]$-basis of $\operatorname{Der}_{\mathbb{C}} \mathbb{C}[X]$.

\subsection{The Jacobian Conjecture and Kernel Conjecture}

Let $F=\left(F_{1}, \ldots, F_{n}\right)$ be a polynomial map with $\operatorname{det}(J F) \in \mathbb{C}^{*}$ and assume that the Jacobian Conjecture is true. Then $\mathbb{C}\left[X_{1}, \ldots, X_{n}\right]=\mathbb{C}\left[F_{1}, \ldots, F_{n}\right]$ and hence

$$
\operatorname{ker}\left(\frac{\partial}{\partial F_{n}}, \mathbb{C}[X]\right)=\operatorname{ker}\left(\frac{\partial}{\partial F_{n}}, \mathbb{C}\left[F_{1}, \ldots, F_{n}\right]\right)=\mathbb{C}\left[F_{1}, \ldots, F_{n-1}\right]
$$

This leads us to

Conjecture 2.4 (Kernel Conjecture $(K C(n)))-$ If $\operatorname{det}(J F) \in \mathbb{C}^{*}$, then

$$
\operatorname{ker}\left(\frac{\partial}{\partial F_{n}}, \mathbb{C}[X]\right)=\mathbb{C}\left[F_{1}, \ldots, F_{n-1}\right] \text {. }
$$

The observation above then states that

$$
J C(n) \text { implies } K C(n), \text { for all } n \geq 1
$$

Conversely we have

Proposition $2.5-K C(n+1)$ implies $J C(n)$ for all $n \geq 1$.

Proof. Let $F=\left(F_{1}, \ldots, F_{n}\right): \mathbb{C}^{n} \rightarrow \mathbb{C}^{n}$ with $\operatorname{det}(J F) \in \mathbb{C}^{*}$. We need to show that $\mathbb{C}\left[F_{1}, \ldots, F_{n}\right]=\mathbb{C}\left[X_{1}, \ldots, X_{n}\right]$. Therefore put $\tilde{F}:=\left(F, X_{n+1}\right): \mathbb{C}^{n+1} \rightarrow \mathbb{C}^{n+1}$. Then $\operatorname{det}(J \tilde{F}) \in \mathbb{C}^{*}$. So by $K C(n+1)$ we get

$$
\operatorname{ker}\left(\frac{\partial}{\partial \tilde{F}_{n+1}}, \mathbb{C}\left[X_{1}, \ldots, X_{n+1}\right]\right)=\mathbb{C}\left[F_{1}, \ldots, F_{n}\right] \text {. }
$$

However $\frac{\partial}{\partial \tilde{F}_{n+1}}=\frac{\partial}{\partial X_{n+1}}$ (since they coincide on $X_{n+1}$ and on each $F_{i}$, they coincide on $\mathbb{C}\left(F_{1}, \ldots, F_{n}, X_{n+1}\right)$ and consequently on $\mathbb{C}\left(X_{1}, \ldots, X_{n+1}\right)$ which is an algebraic extension of $\left.\mathbb{C}\left(F_{1}, \ldots, F_{n}, X_{n+1}\right)\right)$. So

$$
\begin{aligned}
\operatorname{ker}\left(\frac{\partial}{\partial \tilde{F}_{n+1}}, \mathbb{C}\left[X_{1}, \ldots, X_{n+1}\right]\right) & =\operatorname{ker}\left(\frac{\partial}{\partial X_{n+1}}, \mathbb{C}\left[X_{1}, \ldots, X_{n+1}\right]\right) \\
& =\mathbb{C}\left[X_{1}, \ldots, X_{n}\right] .
\end{aligned}
$$


Combining this with $(10)$ we get $\mathbb{C}\left[F_{1}, \ldots, F_{n}\right]=\mathbb{C}\left[X_{1}, \ldots, X_{n}\right]$.

So to study the Jacobian Conjecture we can as well study the Kernel Conjecture. Also we may assume that $n \geq 2$, since $K C(1)$ is obvious. This leads us to the following more general question:

Question 2.6 - Let $n \geq 2$ and $D$ be any non-zero derivation on $k\left[X_{1}, \ldots, X_{n}\right]$, where $k$ is a field with $\operatorname{char}(k)=0$. Does it follow that $k[X]^{D}:=\operatorname{ker}(D, k[X])$ is a polynomial ring in $n-1$ variables?

The answer to this question is obviously no if $n \geq 3$ : just take $D=X_{1} \partial_{1}+$ $\cdots+X_{n} \partial_{n}$ and observe that $D\left(c X_{1}^{i_{1}} \cdots X_{n}^{i_{n}}\right)=c\left(i_{1}+\cdots+i_{n}\right) X_{1}^{i_{1}} \cdots X_{n}^{i_{n}}$, for all $i_{1}, \ldots, i_{n} \geq 0$ and all $c \in k$. This implies that $k[X]^{D}=k$.

In fact if $n \geq 3$ there are all kind of possible kernels.

Proposition 2.7 (Nowicki, Strelcyn, [53]) - Let $n \geq 3$ and $r \geq 0$. Then there exists a $k$-derivation $D$ on $k[X]$ such that the minimal number of generators of $k[X]^{D}$ is equal to $r$ (if $1 \leq r<n$, take $D=X_{r+1} \partial_{r+1}+\cdots+X_{n} \partial_{n}$ and if $r \geq n$ take $\left.D=X_{1} \partial_{1}+X_{2} \partial_{2}+(r-n+2) X_{3} \partial_{3}\right)$.

However if $n=2$ the situation is much better. This case will be studied in the next section.

\subsection{The kernel of a derivation: the case $n=2$}

The main result of this section is

Theorem 2.8 (Nagata, Nowicki, [50]) - Let $k$ be a field with $\operatorname{char}(k)=0$. Then there exists a polynomial $f$ in $k[X, Y]$ with $k[X, Y]^{D}=k[f]$.

This result is based on two highly non-trivial results. The first result is due to Zariski and gives a partial answer to

Problem 2.9 (Hilbert 14-th) - Let $k$ be a field and let $L$ be a subfield of $k\left(X_{1}, \ldots, X_{n}\right)$ containing $k$. Is $L \cap k\left[X_{1}, \ldots, X_{n}\right]$ a finitely generated $k$-algebra?

Theorem 2.10 (Zariski, [64]) - If $\operatorname{trdeg}_{k}(L) \leq 2$, then the answer is yes.

Corollary 2.11 (Nagata, Nowicki, [50]) - If $D$ is a non-trivial derivation on $k\left[X_{1}, \ldots, X_{n}\right]$, then $k[X]^{D}$ is a finitely generated $k$-algebra if $n \leq 3$.

Proof. One easily verifies that $\operatorname{trdeg}_{k}\left(Q\left(k[X]^{D}\right)\right) \leq n-1$ (where $Q($.$) denotes the$ quotient field of (.)). Since

$$
k[X]^{D}=Q\left(k[X]^{D}\right) \cap k[X]
$$

the result follows from Zariski's theorem.

The second ingredient in the proof of theorem 2.8 is a beautiful characterization of a polynomial ring in one variable over a field due to Zaks. 
Theorem 2.12 (Zaks, [63]) - Let $k$ be a field. If $R$ is a Dedekind subring of $k\left[X_{1}, \ldots, X_{n}\right]$ containing $k$, then there exists a polynomial $f \in k\left[X_{1}, \ldots, X_{n}\right]$ such that $R=k[f]$.

Now we are able to give

Proof of Theorem 2.8. Put $R=k[X, Y]^{D}$ and put $s=\operatorname{trdeg}_{k}(Q(R))$. Then $s \leq 1$. If $s=0$ then each element of $k[X, Y]^{D}$ is algebraic over $k$ and hence $R=k$ (since the only elements of $k(X, Y)$ which are algebraic over $k$ are the elements of $k)$. So $R=k[f]$ with $f=1$ for example. Now assume $s=1$. By corollary $2.11 R$ is a finitely generated $k$-algebra. So $R$ is a noetherian domain of dimension one. Finally one easily verifies that $k[X, Y]^{D}$ is integrally closed in $k[X, Y]$ and hence in $k(X, Y)$. So $k[X, Y]^{D}$ is integrally closed. Consequently $R$ is a Dedekind subring of $k[X, Y]$ containing $k$. Then apply theorem 2.12 .

\subsection{Consequences of theorem 2.8}

The aim of this section is to demonstrate the importance of theorem 2.8 , i.e. we will show that it implies $K C(2)$ and how it implies some partial results concerning the two-dimensional Jacobian Conjecture.

Proposition $2.13-K C(2)$ is true.

Proof. By theorem $2.8 \operatorname{ker}\left(\frac{\partial}{\partial F_{2}}, \mathbb{C}\left[X_{1}, X_{2}\right]\right)=\mathbb{C}[f]$ for some $f \in \mathbb{C}\left[X_{1}, X_{2}\right]$. Since we have $\frac{\partial}{\partial F_{2}}\left(F_{1}\right)=0$ we deduce that $F_{1}=g(f)$ for some non-zero polynomial $g(T) \in \mathbb{C}[T]$. Apply $\frac{\partial}{\partial F_{2}}$ to this equation and observe that $\frac{\partial}{\partial F_{1}} \mathbb{C}[f] \subset \mathbb{C}[f]$ since $\frac{\partial}{\partial F_{1}}$ and $\frac{\partial}{\partial F_{2}}$ commute. Consequently $1=g^{\prime}(f) \frac{\partial}{\partial F_{1}} f$ in $\mathbb{C}[f]$, so $g^{\prime}(f) \in \mathbb{C}^{*}$, i.e. $F_{1}=g(f)=\lambda f+\mu$ for some $\lambda, \mu \in \mathbb{C}, \lambda \neq 0$. Consequently $\mathbb{C}[f]=\mathbb{C}\left[F_{1}\right]$.

Now we will show the importance of theorem 2.8 in connection with the twodimensional Jacobian Conjecture. The results described in the remainder of this section are all taken from the elegant paper [46] of Nagata.

We assume that the reader is familiar with the concept of Newton Polygon and that of radial similarity (we refer to [46] and the paper [8] of Cheng and Wang, which contain all necessary definitions).

Theorem 2.14 - Let $k$ be a field with $\operatorname{char}(k)=0$ and $f, g \in k[X, Y]$. If $\operatorname{det}(J(f, g))=0$, then the Newton polygon of $f$ is similar to the one of $g$ with the origin as center of similarity and with ratio $\operatorname{deg}(f): \operatorname{deg}(g)$.

Proof. Let $m=\operatorname{deg}(f)$ and $n=\operatorname{deg}(g)$ and let $f=f_{m}+f_{m-1}+\cdots+f_{0}$, $g=g_{n}+g_{n-1}+\cdots+g_{0}$ be the homogeneous decompositions of $f$ and $g$. Since $\operatorname{det}(J(f, g))=0$ we get $\operatorname{det}\left(J\left(f_{m}, g_{n}\right)\right)=0$. So if $D=\frac{\partial}{\partial Y}\left(f_{m}\right) \frac{\partial}{\partial X}-\frac{\partial}{\partial X}\left(f_{m}\right) \frac{\partial}{\partial Y}$ then both $f_{m}, g_{n} \in k[X, Y]^{D}$. By theorem $2.8 k[X, Y]^{D}=k[h]$ for some $h \in k[X, Y]$. Since 
$f_{m}$ and $g_{n}$ are homogeneous and belong to $k[h]$ it follows that $h$ is homogeneous and consequently $f_{m}=a h^{m^{\prime}}, g_{n}=b h^{n^{\prime}}$ for some $a, b \in k$ and $m^{\prime}, n^{\prime} \in \mathbb{N}$. So $\operatorname{Supp}\left(f_{m}\right)$ is similar to $\operatorname{Supp}\left(g_{n}\right)$ with the origin as center of similarity and ratio $m: n$. Take one end of $\operatorname{Supp}\left(f_{m}\right)$ and the corresponding end of $\operatorname{Supp}\left(g_{n}\right)$, say they are the points $(a, b)$ resp. $(c, d)$. So they satisfy $n(a, b)=m(c, d)$. Now take one direction $(p, q)$ such that the leading $(p, q)$-form $f^{\prime}, g^{\prime}$ of $f, g$ has terms $X^{a} Y^{b}, X^{c} Y^{d}$ with non-zero coefficients respectively. Since $\operatorname{det}\left(J\left(f^{\prime}, g^{\prime}\right)\right)=0$ we deduce as before that $\operatorname{Supp}\left(f^{\prime}\right)$ and $\operatorname{Supp}\left(g^{\prime}\right)$ are similar with the origins as center of similarity. Since the points $(a, b)$ and $(c, d)$ are on $\operatorname{Supp}\left(f^{\prime}\right), \operatorname{Supp}\left(g^{\prime}\right)$ respectively we see that the ratio is $m: n$ again! This argument can be applied to any neighbouring edge succesively.

The proof given above, supplemented with some simple degree argument and proposition 2.16 below, can be used to give a very short and simple proof of the following result (cf. [46]).

Theorem 2.15 - Let $k$ be a field with $\operatorname{char}(k)=0$ and $f, g \in k[X, Y]$. If $\operatorname{det}(J(f, g)) \in k^{*}$ and $\operatorname{deg}(f), \operatorname{deg}(g)>1$ then the Newton polygon of $f$ is similar to the one of $g$ with the origin as center of similarity and with ratio $\operatorname{deg}(f): \operatorname{deg}(g)$.

An immediate consequence of this theorem is that under the hypothesis of the theorem both the $X$-axis and the $Y$-axis contain points of $\operatorname{Supp}(f)$ as well as of $\operatorname{Supp}(g)$. More precisely, if for a polynomial $h \in k[X, Y]$ we define $t_{x}(h)$ and $t_{y}(h)$ by

$$
\begin{aligned}
& t_{x}(h)=\max \{s \mid(s, 0) \in \operatorname{Supp}(h) \cup\{(0,0)\}\} \\
& t_{y}(h)=\max \{s \mid(0, s) \in \operatorname{Supp}(h) \cup\{(0,0)\}\}
\end{aligned}
$$

then we have

Proposition 2.16 - If $\operatorname{det}(J(f, g)) \in k^{*}$ and $\operatorname{deg}(f), \operatorname{deg}(g)>1$ then $t_{x}(f), t_{y}(f)$, $t_{x}(g)$ and $t_{y}(g)$ are all positive.

Proof. Since the linear part of $(f, g)$ is invertible $(1,0)$ and $(0,1)$ belong to $\operatorname{Supp}(f) \cup \operatorname{Supp}(g)$. Then apply theorem 2.15.

Remark 2.17. In Nagata's paper [46] proposition 2.16 is proved independently of theorem 2.15 (only using a simple degree argument and theorem 2.8).

Corollary 2.18 (Magnus, [40]) - Let $\operatorname{char}(k)=0$ and $\operatorname{det}(J(f, g)) \in k^{*}$. If $\operatorname{gcd}(\operatorname{deg}(f), \operatorname{deg}(g))=1$ then $k[f, g]=k[X, Y]$ i.e. $F=(f, g)$ is an automorphism.

Proof. If one of $\operatorname{deg}(f)$ or $\operatorname{deg}(g)$ is 1 , then one easily verifies that $k[f, g]=k[X, Y]$. So we may assume that $m:=\operatorname{deg}(f)$ and $n:=\operatorname{deg}(g)$ are both bigger than one. We will derive a contradiction. Therefore write $f=f_{m}+\cdots+f_{0}$ and $g=g_{n}+\cdots+g_{0}$, the homogeneous decomposition. Then as in the proof of theorem 2.14 there exists 
a homogeneous polynomial $h$ such that $f_{m}=a h^{m^{\prime}}, g_{n}=b h^{n^{\prime}}$ with $a, b \in k^{*}$, $n^{\prime}, m^{\prime} \in \mathbb{N}$. Since $\operatorname{gcd}(n, m)=1$ we deduce that $\operatorname{deg}(h)=1$. Obviously we may assume that $h=X$. By theorem 2.15 we have $t_{y}(f) n=t_{y}(g) m$. So $t_{y}(f)$ is divisible by $m$. However $t_{y}(f)>0$ (by proposition 2.16) and $t_{y}(f)<m$, since $f_{m}=a X^{m}$ $(h=X !)$, a contradiction.

Corollary 2.19 (Magnus, [40]) - If $\operatorname{det}(J(f, g)) \in k^{*}$ and $\operatorname{deg}(f)$ or $\operatorname{deg}(g)$ is a prime number, then $k[f, g]=k[X, Y]$.

Proof. Suppose $\operatorname{deg}(f)=p$ is a prime number. We use induction on $\operatorname{deg}(g)$. In case $\operatorname{gcd}(\operatorname{deg}(f), \operatorname{deg}(g))=1$ we are done by corollary 2.18 . So in particular we are done if $\operatorname{deg}(g)<\operatorname{deg}(f)$. So we may assume that $\operatorname{deg}(g) \geq \operatorname{deg}(f)$ and hence (since $\operatorname{deg}(f)$ is prime) that $p=\operatorname{deg}(f) \operatorname{divides} \operatorname{deg}(g)$. Again we have $f_{m}=a h^{m^{\prime}}, g_{n}=b h^{n^{\prime}}$ for some $h \in k[X, Y]$. So $m^{\prime}$ divides $n^{\prime}$ i.e. $n^{\prime}=d m^{\prime}$ for some $d \in \mathbb{N}$. Hence if we put $g_{1}:=g-\lambda f^{d}$ where $b-\lambda a^{d}=0$, then we have $\operatorname{deg}\left(g_{1}\right)<\operatorname{deg}(g)$. Obviously $\operatorname{det}\left(J\left(f, g_{1}\right)\right)=\operatorname{det}(J(f, g)) \in k^{*}$. So $k\left[f, g_{1}\right]=k[X, Y]$ by the induction hypothesis. Consequently $k[f, g]=k\left[f, g_{1}\right]=k[X, Y]$.

Remark 2.20. In [46] Nagata gives an improvement of corollary 2.18: namely the assumption $d=\operatorname{gcd}(\operatorname{deg}(f), \operatorname{deg}(g))=1$ is replaced by $d \leq 8$.

To formulate the last result of this section we need to recall the celebrated Abhyankar-Moh theorem (a short proof of it using knot-theory was given by Rudolph in [55]):

Theorem 2.21 (Abhyankar-Moh, [2]) - Let $k$ be an algebraically closed field of characteristic zero. Let $\gamma: k \rightarrow k^{2}$ be an embedding i.e. $\gamma$ is injective and $\gamma^{\prime}(t) \neq 0$ for all $t \in k$. Then there exists a polynomial automorphism $H: k^{2} \rightarrow k^{2}$ such that $\gamma(t)=H(t, 0)$, for all $t \in k$.

Now we are able to prove

Theorem 2.22 (Gwoździewicz, [30]) - Let $k$ be an algebraically closed field of characteristic zero. Let $\operatorname{det}(J(f, g)) \in k^{*}$. If $F=(f, g): k^{2} \rightarrow k^{2}$ is injective on one line $\ell \subset k^{2}$ then $F$ is an automorphism!

Proof. We may assume that $\ell$ has the equation $Y=0$. Define $\gamma: k \rightarrow k^{2}$ by $\gamma(x)=F(x, 0)$. So $\gamma$ is injective and $\operatorname{det}(J(f, g)) \in k^{*}$ implies that $\gamma^{\prime}(x) \neq 0$ for all $x \in k$. So by theorem $2.21 \gamma(x)=H(x, 0)$ for some automorphism $H$. Put $G=H^{-1} \circ F$. Then $\operatorname{det}(J G) \in k^{*}$ and $G(x, 0)=(x, 0)$. Write $G=\left(g_{1}, g_{2}\right)$. If both $\operatorname{deg}\left(g_{1}\right)>1$ and $\operatorname{deg}\left(g_{2}\right)>1$ then by proposition $2.16 t_{x}\left(g_{2}\right)>0$, contradicting $g_{2}(x, 0)=0$. So either $\operatorname{deg}\left(g_{1}\right)$ or $\operatorname{deg}\left(g_{2}\right) \leq 1$, in which case one easily deduces that $G$ is a polynomial automorphism, hence so is $F(=H \circ G)$. 


\subsection{The kernel of a derivation: the case $n>2$}

Now we consider the case $n>2$. Here the situation is completely different from the $n=2$ case. We already saw in proposition 2.7 that if $D$ is any derivation on $k\left[X_{1}, \ldots, X_{n}\right]$ and $n \geq 3$, then $k[X]^{D}$ need not be a polynomial ring in $n-1$ variables. However the situation is worse: in 1958 Nagata in [47] gave a counterexample to Hilbert 14-th problem for the case $n=32$. In other words, he constructed a subfield $L$ of $\mathbb{C}\left(X_{1}, \ldots, X_{32}\right)$ such that $L \cap \mathbb{C}\left[X_{1}, \ldots, X_{32}\right]$ is not a finitely generated $\mathbb{C}$-algebra. In 1991 Derksen in [14] constructed a derivation $D$ on $\mathbb{C}\left[X_{1}, \ldots, X_{32}\right]$ such that $\mathbb{C}[X]^{D}=L \cap \mathbb{C}\left[X_{1}, \ldots, X_{32}\right]$ and hence $\mathbb{C}[X]^{D}$ is not a finitely generated $\mathbb{C}$-algebra.

More recently, Roberts in [54] 1990 gave a new counterexample to Hilbert 14-th in dimension 7. As a consequence one can deduce:

Proposition 2.23 - For each $t \geq 2$ is the kernel of the derivation

$$
D=X^{t+1} \partial_{S}+Y^{t+1} \partial_{T}+Z^{t+1} \partial_{U}+(X Y Z)^{t} \partial_{V}
$$

on $\mathbb{C}[X, Y, Z, S, T, U, V]$ not a finitely generated $\mathbb{C}$-algebra.

Remark 2.24. The case $t=2$ was treated with a new proof by Deveney and Finston in [15].

Remark 2.25. In [25] the authors introduce and study so-called elementary derivations i.e. derivations of the form

$$
D=a_{1}\left(X_{1}, \ldots, X_{n}\right) \frac{\partial}{\partial Y_{1}}+\cdots+a_{m}\left(X_{1}, \ldots, X_{n}\right) \frac{\partial}{\partial Y_{m}}
$$

on the $n+m$-variable polynomial ring $k\left[X_{1}, \ldots, X_{n}, Y_{1}, \ldots, Y_{m}\right]$, where $k$ is a field with $\operatorname{char} k=0$. They show that if $n \leq 2$ or $m \leq 2$ the kernel of $D$ is finitely generated over $k$.

It is still an open problem if in case $n=3, m=3$ there exists an elementary derivation which kernel is not finitely generated. In [25] some candidate counterexamples are constructed.

\section{Problems related to the Jacobian Conjecture}

\subsection{Cancellation problems}

In this section we discuss several cancellation problems and their relationship with the Jacobian Conjecture.

Problem 3.1 (Biregular Cancellation problem) - Given two affine varieties $Y$ and $Z$ over $\mathbb{C}$. Suppose that for some $n \in \mathbb{N} Y \times \mathbb{C}^{n}$ is isomorphic with $Z \times \mathbb{C}^{n}$. Does it follow that $Y$ is isomorphic to $Z$ ? 
Problem 3.2 (Cancellation problem) - Let $m \in \mathbb{N}$ and $Z=\mathbb{C}^{m}$. Same question.

Problem 3.3 (Birational Cancellation problem) - Let $Y$ and $Z$ be two irreducible varieties over $\mathbb{C}$. Suppose that for some $n \in \mathbb{N} Y \times \mathbb{P}^{n}$ is birationally isomorphic to $Z \times \mathbb{P}^{n}$. Does it follow that $Y$ is birationally isomorphic to $Z$ ? $\left(\mathbb{P}^{n}\right.$ denotes the $n$-dimensional complex projective space.)

Problem 3.4 (Rational Cancellation problem) - Let $m \in \mathbb{N}$ and $Z=\mathbb{P}^{m}$. Same question.

Before we discuss the present status of these cancellation problems, let us first describe in which sense the Jacobian Conjecture is related to the Cancellation problem. Therefore we first formulate the Cancellation problem $(C P)$ in algebraic terms. Ofcourse the $C P$ is equivalent to the question if $Y \times \mathbb{C} \simeq \mathbb{C}^{m}$ implies that $Y \simeq \mathbb{C}^{m-1}$. Therefore we get:

Problem 3.5 (Cancellation problem (algebraic form)) -

Let $\mathbb{C}\left[X_{1}, \ldots, X_{n}\right]=A[T]$ be a polynomial ring in one variable $T$ over a $\mathbb{C}$ algebra $A$. Does it follow that $A$ is a polynomial ring in $n-1$ variables over $\mathbb{C}$ i.e. $A=\mathbb{C}\left[F_{1}, \ldots, F_{n-1}\right]$ for some $F_{i} \in \mathbb{C}[X]$, algebraic independent over $\mathbb{C}$ ?

We saw in (9) and proposition 2.5 that the Jacobian Conjecture is equivalent to the Kernel Conjecture. Now we will show that also the $C P$ can be reformulated as a kernel problem, which clearly shows that both the Jacobian Conjecture and the Cancellation problem have some common roots. Therefore recall that a derivation $D$ on a ring $R$ is called locally nilpotent if for every $r \in R$ there exists an integer $m$ such that $D^{m} r=0$. To prove the desired equivalence we need the following well-known result (cf. [59]).

Proposition 3.6 - Let $R$ be a $\mathbb{Q}$-algebra and $D: R \rightarrow R$ a locally nilpotent derivation on $R$ such that $D s=1$ for some $s \in R$ ( $s$ is called a slice). Then $R=R^{D}[s]$ i.e. $R$ is a polynomial ring in $s$ over $R^{D}$.

Problem 3.7 (2-nd Kernel problem) - Let $D$ be a locally nilpotent derivation on $\mathbb{C}[X]$ having a slice. Does it follow that $\mathbb{C}[X]^{D}=\mathbb{C}\left[F_{1}, \ldots, F_{n-1}\right]$ for some $F_{i} \in \mathbb{C}[X]$ algebraically independent over $\mathbb{C}$ ?

Proposition 3.8 - The Cancellation problem is equivalent to the 2-nd Kernel problem.

Proof. Let $D$ be a locally nilpotent derivation on $\mathbb{C}[X]$ with a slice $s$. Then by proposition $3.6 \mathbb{C}[X]=\mathbb{C}[X]^{D}[s]$. So if the Cancellation problem is true, then $\operatorname{ker}(D, \mathbb{C}[X])=\mathbb{C}[X]^{D}=\mathbb{C}\left[F_{1}, \ldots, F_{n-1}\right]$ for some $F_{i} \in \mathbb{C}[X]$ algebraically independent over $\mathbb{C}$.

Conversely, let $\mathbb{C}[X]=A[T]$. Then the derivation $\frac{d}{d T}$ is locally nilpotent on $A[T]=\mathbb{C}[X]$ and has a slice, $T$. Furthermore $\operatorname{ker}\left(\frac{d}{d T}, \mathbb{C}[X]\right)=A$. So if the 2- 
nd Kernel problem is true, then $A=\mathbb{C}\left[F_{1}, \ldots, F_{n-1}\right]$ for some $F_{i}$ algebraically independent over $\mathbb{C}$, which shows that the Cancellation problem is true.

So both the Jacobian Conjecture as well as the Cancellation problem ask if the kernel of a certain type of derivation on $\mathbb{C}\left[X_{1}, \ldots, X_{n}\right]$ is a polynomial ring in $n-1$ variables.

Now let's return to the cancellation problems described above. (For more details we refer to the paper [36] by Kraft, the papers [33] and [34] by Kang, the paper [57] by Sugie and the paper [45] by Miyanishi and Sugie.)

\subsubsection{The Biregular Cancellation problem}

In algebraic form the biregular cancellation problem reads as follows.

Problem 3.9 (Biregular Cancellation problem (algebraic form)) - Let $A$ and $B$ be affine domains over $\mathbb{C}$. Suppose that $A\left[X_{1}, \ldots, X_{n}\right]=B\left[Y_{1}, \ldots, Y_{n}\right]$ which are polynomial rings over $A$ respectively $B$. Does it follow that $A$ is isomorphic to $B$ over $k$ ?

Here we have a similar phenomenon as we met in section 2.5 where we considered Hilbert 14-th problem and the kernel problem. Both had an affirmative answer in low dimension but a negative answer in high dimension.

Theorem 3.10 (Abhyankar, Eakin, Heinzer, [1]) - Let $k$ be a field, $A$ and $B$ commutative integral domains containing $k$. Suppose that $A\left[X_{1}, \ldots, X_{n}\right]=B\left[Y_{1}, \ldots, Y_{n}\right]$ and $\operatorname{trdeg}_{k}(Q(A)) \leq 1$. Then either $A=B$ or $A$ and $B$ are isomorphic to a polynomial ring over $k_{0}$, where $k_{0}$ is $A \cap B$, is the algebraic closure of $k$ in $Q(A)$, is the algebraic closure of $k$ in $Q(B)$.

However if $\operatorname{trdeg}_{k}(Q(A)) \geq 2$ the answer is no in general as is shown by the following example due to Danielewski (cf. [10]).

Theorem 3.11 - For $n \geq 1$ let $Y_{n} \subset \mathbb{C}^{3}$ be the closed subvariety defined by the equation $x^{n} y+z^{2}=1$. Then all varieties $Y_{n} \times \mathbb{C}$ are isomorphic. However the topological spaces $Y_{n}$ are all of different homotopy type.

\subsubsection{The Birational Cancellation problem}

Problem 3.12 (Birational Cancellation problem (algebraic form)) -

Let $K_{1}$ and $K_{2}$ be finitely generated field extensions of $\mathbb{C}$. Suppose that $K_{1}\left(X_{1}, \ldots, X_{n}\right)=K_{2}\left(Y_{1}, \ldots, Y_{n}\right)$. Does it follow that $K_{1}$ and $K_{2}$ are $\mathbb{C}$ isomorphic?

Theorem 3.13 (cf. [33], [34]) - Let $k$ be any field, $K_{1}$ and $K_{2}$ finitely generated field extensions of $k$. Suppose that $K_{1}\left(X_{1}, \ldots, X_{n}\right)=K_{2}\left(Y_{1}, \ldots, Y_{n}\right)$. 
1. If $\operatorname{trdeg}_{k}\left(K_{i}\right) \leq 1, i=1,2$, then $K_{1} \simeq K_{2}$ over $k$.

2. If $k$ is an algebraically closed field of characteristic zero and $\operatorname{trdeg}_{k}\left(K_{i}\right)=2$, $i=1,2$, then $K_{1} \simeq K_{2}$ over $k$.

However if $\operatorname{trdeg}_{k}(K)=3$ we have the following counterexample to the rational (and hence to the birational) cancellation problem.

Theorem 3.14 - ([5], Beauville, Colliot-Thélène, Sansuc, SwinnertonDyer) Let $k$ be an algebraically closed field with $\operatorname{char}(K) \neq 2$. Let

$$
K=Q\left(k[X, Y, Z, W] /\left(X^{2}-a(W) Y^{2}-f(W, Z)\right)\right.
$$

where $f(W, Z) \in k[W, Z]$ is irreducible of degree 3 in $Z$ and

$$
a(W)=\operatorname{disc}_{Z}(f(W, Z)) \in k[W] \backslash\{0\}
$$

is squarefree of degree $\geq 5$. Then

1. $K$ is not rational over $k$.

2. $K\left(T_{1}, T_{2}, T_{3}\right)$ is $k$-isomorphic to a rational function field in six variables over $k$.

\subsubsection{The Cancellation problem}

In theorem 3.11 we saw that if $\operatorname{dim}(Y)=\operatorname{dim}(Z)=2$ then the answer to the biregular cancellation problem is negative. However if we consider the cancellation problem in dimension two i.e. $Z=\mathbb{C}^{2}$, then we have

Theorem 3.15 (Fujita, Miyanishi, Sugie, [27], [45], [57]) - If $Y \times \mathbb{C}^{n} \simeq \mathbb{C}^{n+2}$, then $Y \simeq \mathbb{C}^{2}$.

In fact this result is a consequence of a beautiful characterization of the affine plane $\mathbb{C}^{2}$ (due to the above mentioned work of Fujita, Miyanishi and Sugie):

Theorem 3.16 - Let $Y$ be a smooth factorial affine surface. If there is a dominant morphism $\varphi: \mathbb{C}^{n} \rightarrow Y$ for some $n \in \mathbb{N}$, then $Y$ is isomorphic to $\mathbb{C}^{2}$. (Algebraically: let $R$ be a smooth affine subring of $\mathbb{C}\left[X_{1}, \ldots, X_{n}\right]$ of dimension two. If $R$ is a U.F.D. then $R=\mathbb{C}\left[F_{1}, F_{2}\right]$ for some $F_{i} \in \mathbb{C}[X]$ algebraically independent over $\mathbb{C}$.)

Now the next case is $\operatorname{dim}(Y)=3$. So the first question is: what happens to theorem 3.16 if we assume that $\operatorname{dim}(Y)=3$ ? The answer is: the analogues result of this theorem is false if $\operatorname{dim}(Y)=3$.

Counterexample $3.17-$ (cf. [37, example 1]) The surface $Y \subset \mathbb{C}^{4}$ given by the equation $x+x^{2} y+z^{2}+t^{3}=0$ is factorial and there is a dominant morphism $\varphi: \mathbb{C}^{3} \rightarrow Y$. Furthermore $Y$ is smooth and diffeomorphic to $\mathbb{C}^{3}$. However it was recently shown by Makar-Limanov in [41] that $Y$ is not algebraically isomorphic to 
$\mathbb{C}^{3}$. In [13] Harm Derksen gives a much shorter proof of this result. His basic idea is to consider the coordinate ring $R$ of the surface $Y$ and to show that $C(R):=$ the $\mathbb{C}$-subalgebra of $R$ generated by the subrings $R^{D}$, where $D$ runs through all nontrivial locally nilpotent derivations of $R$, is strictly smaller than $R$. This is obviously impossible if $R=\mathbb{C}[X, Y, Z]$ (for in that case the kernel of $\frac{\partial}{\partial X}, \frac{\partial}{\partial Y}$ and $\frac{\partial}{\partial Z}$ are $\mathbb{C}[Y, Z], \mathbb{C}[X, Z]$ and $\mathbb{C}[X, Y]$ respectively and they generate $\mathbb{C}[X, Y, Z])$.

\subsubsection{Summarizing}

There is strong evidence to believe that also the cancellation problem is false if $n$ is large enough. Since both the cancellation problem and the Jacobian problem are problems of the same type (both are kernel problems) these arguments also support the believe that the Jacobian Conjecture is false too.

\subsection{Linearization problems}

There are several papers concerning linearization problems, in particular the linearization conjecture of Kambayashi ([32], 1979) which asserts that every action of a complex algebraic reductive group on $\mathbb{C}^{n}$ is linearizable, has attracted much attention. Several partial results are known. It was finally answered in the negative by Schwarz in [56]. For more details (and references) we refer to the survey paper of Kraft [36]. In this paper we only mention some linearization problems which arose in connection with the Jacobian Conjecture. So the questions we consider are of the form

Question 3.18 - Which polynomial automorphism $F: \mathbb{C}^{n} \rightarrow \mathbb{C}^{n}$ are linearizable i.e. are such that there exists a polynomial automorphism $\varphi: \mathbb{C}^{n} \rightarrow \mathbb{C}^{n}$ such that $\varphi^{-1} F \varphi$ is linear?

Before we describe the relationship between the Jacobian Conjecture and linearization problems let us briefly point out that also the cancellation problem is related to a linearization problem.

Proposition 3.19 - If every polynomial map $F: \mathbb{C}^{n} \rightarrow \mathbb{C}^{n}$ satisfying $F^{2}=I$ is linearizable, then the cancellation problem is true.

Proof. 1. Observe that if a map $F$ is linearizable then its fixpoint set $\operatorname{Fix}(F)$ is isomorphic to some $\mathbb{C}^{d}\left(\mathbb{C}^{0}:=\{0\}\right)$.

2. Now suppose that $Y \times \mathbb{C} \simeq \mathbb{C}^{n}$. Then the automorphism $F: \mathbb{C}^{n} \rightarrow \mathbb{C}^{n}$ given by $F(y, t)=(y,-t)$ satisfies $F^{2}=I$ and $\operatorname{Fix}(F) \simeq Y$. So by 1 and the hypothesis $Y \simeq \mathbb{C}^{d}$, whence $Y \simeq \mathbb{C}^{n-1}$. 
Now let's return to the Jacobian Conjecture. The connection between the Jacobian Conjecture and linearization problems comes from an attempt of Deng, Meisters and Zampieri to prove the Jacobian Conjecture. In [12] they proved that if $\operatorname{det}(J F) \in \mathbb{C}^{*}$ then for all $s \in \mathbb{C}$ with $|s|$ large the map $s F$ is linearizable to $s J F(0) X$ by means of a locally analytic map $\varphi_{s}$ which inverse is an entire function. Their aim was to prove that $\varphi_{s}$ is entire which would imply that $s F$ and hence $F$ is injective, so the Jacobian Conjecture would follow.

However they were not able to prove the entireness of $\varphi_{s}$. (In fact it was recently shown by van den Essen and Hubbers in [24] that $\varphi_{s}$ need not be entire!) So Meisters started to look at examples of $F$ 's of the form $X+H, H$ cubic homogeneous. In all the examples he computed it turned out that the $\varphi_{s}$ was even better as expected: they were polynomial automorphisms! This lead him to the following conjecture

Conjecture 3.20 (Meisters' Linearization Conjecture (cf. [43])) -

Let $F=X+H$ with $H$ cubic homogeneous and $J H$ nilpotent (or equivalently $\left.\operatorname{det}(J F) \in \mathbb{C}^{*}\right)$, then for almost all $s \in \mathbb{C}$ except a finite number of roots of unity) there exists a polynomial automorphism $\varphi_{s}$ such that $\varphi_{s}^{-1} \circ s F \circ \varphi_{s}=s X$.

It turned out that this conjecture is true if $n \leq 3$ (cf. [21]) and false if $n \geq 4$ :

Theorem 3.21 (Van den Essen, [21]) - Let $d(X)=X_{3} X_{1}+X_{4} X_{2}$. For every $n \geq 4$ the polynomial map

$$
F=\left(X_{1}+X_{4} d(X), X_{2}-X_{3} d(X), X_{3}+X_{4}^{3}, X_{4}, \ldots, X_{n}\right)
$$

is a counterexample to Meisters' conjecture.

Remark 3.22. In a recent preprint [28] Gorni and Zampieri showed that for each $s \in \mathbb{C} \backslash\{0\},|s| \neq 1$ the map $s F$ admits a global analytic conjugation i.e. there exists an entire map $\varphi_{s}$ such that $\varphi_{s}^{-1} \circ s F \circ \varphi_{s}=s I$. Another proof of this fact was given by Bo Deng in [11]. In fact his very short proof is a consequence of his main result which asserts: let $F: \mathbb{C}^{n} \rightarrow \mathbb{C}^{n}$ be an analytic map on $\mathbb{C}^{n}$ with $F(0)=0$ and such that the eigenvalues of $A:=J F(0)$ have their absolute values strictly between 0 and 1 and have no resonance, then $F$ has an analytic automorphic conjugation to its linear part $A x$ if and only if $F$ is an analytic automorphism of $\mathbb{C}^{n}$ and 0 is a global attractor i.e. $F^{k}(x) \rightarrow 0$ as $k \uparrow \infty$, for all $x \in \mathbb{C}^{n}$. However it was shown in [24] that the following modification of $F$ leads to a counterexample of the original Deng-Meisters-Zampieri Conjecture: let

$$
\tilde{F}=\left(X_{1}+X_{4} d(X)^{2}, X_{3}-X_{2} d(X)^{2}, X_{3}+X_{4}^{m}, X_{4}, \ldots, X_{n}\right),
$$

$m \geq 1$. Then for all $\lambda>1, \lambda \tilde{F}$ is not global analytic linearizable; in fact it is shown that if $a>0$ such that $\lambda a>1$ then $(\lambda \tilde{F})^{k}(a, \ldots, a) \rightarrow \infty$ if $k \uparrow \infty$.

Although Meisters' conjecture is false if $n \geq 4$ it turned out that it is true for a large class of polynomial maps $F$ : 
Definition 3.23 - Let $H: \mathbb{C}^{n} \rightarrow \mathbb{C}^{n}$ be a polynomial map. We say that $J H$ is strongly nilpotent if $J H\left(x_{1}\right) \cdot J H\left(x_{2}\right) \cdots J H\left(x_{n}\right)=0$ for all vectors $x_{1}, x_{2}, \ldots, x_{n} \in$ $\mathbb{C}^{n}$.

Example. If $H$ is an upper-triangular map i.e.

$$
\begin{aligned}
H_{1} & =h_{1}\left(X_{2}, \ldots, X_{n}\right) \\
H_{2} & =h_{2}\left(X_{3}, \ldots, X_{n}\right) \\
& \vdots \\
H_{n-1} & =h_{n-1}\left(X_{n}\right) \\
H_{n} & =0
\end{aligned}
$$

then one esily verifies that $J H$ is upper-triangular and hence strongly nilpotent.

Now we have the following result

Theorem 3.24 (Van den Essen, Hubbers, [23]) - If $F=X+H$ with JH strongly nilpotent, then $s F$ is linearizable for allmost all $s \in \mathbb{C}$ i.e. except a finite number of roots of unity.

The proof of this result consists of two steps. First we show that a map of the form $F=X+H$ is linearly triangulizable (i.e. there exists $T \in \mathrm{GL}_{n}(\mathbb{C})$ such that $T^{-1} H T$ is an upper triangular map) if and only if $J H$ is stongly nilpotent. Then we show that for maps of the form $F=X+H$ with $H$ upper triangular, Meisters' conjecture is true. This is done by induction on $n$ and using a sequence of upper triangular polynomial automorphisms $\varphi$ which are chosen in such a way that at each step the leading monomial (with respect to some ordering) appearing in $H$ is killed.

\subsection{Problems implying the Jacobian Conjecture}

In this section we discuss two open problems which if true would imply the Jacobian Conjecture: the Generalized Dixmier Conjecture and the Markus-Yamabe Conjecture.

\subsubsection{The Generalized Dixmier Conjecture}

Let $k$ be a field of characteristic zero and denote by $A_{n}$ the $n$-th Weyl algebra i.e. $A_{n}=k\left[X_{1}, \ldots, X_{n}, \partial_{1}, \ldots, \partial_{n}\right]$. So we have the relations $\left[\partial_{i}, X_{j}\right]=\delta_{i j}$ and $\left[\partial_{i}, \partial_{j}\right]=\left[X_{i}, X_{j}\right]=0$ for all $i, j$. Each element $P$ in the Weyl algebra can be written uniquely in the form $P=\sum a_{\alpha} \partial^{\alpha}$ and $A_{n}$ is a filtered ring with filtration $F=\left\{A_{n}(v)\right\}_{v \geq 0}$, where $A_{n}(v)$ is the set of operators $\sum a_{\alpha} \partial^{\alpha}$ with $|\alpha| \leq v$ (here $\left.|\alpha|=\alpha_{1}+\cdots+\alpha_{n}\right)$. If we say that $\varphi$ is an endomorphism of $A_{n}$ we always mean that $\varphi$ is $k$-linear. 
Let $\varphi: A_{n} \rightarrow A_{n}$ be a homomorphism. Then obviously $\varphi$ is completely determined by the images of the $X_{i}$ and $\partial_{j}$. Since these images satisfy the same relations as the $X_{i}$ and $\partial_{j}$ one easily deduces:

Proposition 3.25 - Every endomorphism of the Weyl algebra is injective.

Conjecture 3.26 (Generalized Dixmier conjecture) - Each endomorphism of the Weyl algebra is surjective (hence an automorphism).

Remark 3.27. In case $n=1$ this conjecture was formulated in 1968 by Dixmier (cf. [16]). The conjecture is open for all $n \geq 1$.

Proposition 3.28 - The Generalized Dixmier Conjecture implies the Jacobian Conjecture.

Proof. 1. Let $F_{1}, \ldots, F_{n} \in k[X]$ with $\operatorname{det}(J F) \in k^{*}$. Then consider the derivations $\frac{\partial}{\partial F_{1}}, \ldots, \frac{\partial}{\partial F_{n}}$ as defined in (4). Now define a ringhomomorphism $\varphi: A_{n} \rightarrow A_{n}$ by $\varphi\left(X_{i}\right)^{n}=F_{i}$ and $\varphi\left(\partial_{j}\right)=\frac{\partial}{\partial F_{j}}$. So assuming the Generalized Dixmier Conjecture $\varphi$ is surjective.

2. Let $g \in k[X] \subset A_{n}$. Then there exists $P \in A_{n}$ with $g=\varphi(P)$. So $g=\sum a_{\alpha}(F)\left(\frac{\partial}{\partial F}\right)^{\alpha}$. Now apply the operator $g$ to the element $1 \in k[X]$. This gives $g=a_{0}(F) \in k[F]$. So $k[X] \subset k[F]$, implying $k[X]=k[F]$ i.e. $F$ is an automorphism.

Looking at the proof given above we observe that the homomorphism $\varphi$ constructed preserves the filtration $\Gamma$ i.e. $\varphi\left(A_{n}(v)\right) \subset A_{n}(v)$ for all $v \geq 0$; we call such a homomorphism $\Gamma$-preserving. So if we put

Conjecture 3.29 (Weak Dixmier conjecture) - Each Г-preserving endomorphism of $A_{n}$ is surjective.

then the proof of proposition 3.28 gives

Proposition 3.30 - The Weak Dixmier conjecture implies the Jacobian Conjecture.

In fact we have

Theorem 3.31 (Van den Essen, [19]) - The Jacobian Conjecture is equivalent to the Weak Dixmier Conjecture.

Proof. By proposition 3.30 it remains to show that the Jacobian Conjecture implies the Weak Dixmier Conjecture. So assume that the Jacobian Conjecture is true. Let $\varphi$ be a $\Gamma$-preserving endomorphism of $A_{n}$. So $\varphi\left(X_{i}\right) \in k[X]$ and $\varphi\left(\partial_{j}\right) \in A_{n}(1)$, say $\varphi\left(X_{i}\right)=F_{i}, \varphi\left(\partial_{j}\right)=\sum_{k} a_{j k} \partial_{k}+b_{j}$, for some $a_{j k}, b_{j}$ in $k[X]$. From $\left[\varphi\left(\partial_{j}\right), \varphi\left(X_{i}\right)\right]=$ $\delta_{i j}$ we get $\sum a_{j k} \partial_{k}\left(F_{i}\right)=\delta_{j i}$, hence

$$
\left(a_{j k}\right)(J F)^{T}=I_{n}
$$


So $\operatorname{det}(J F) \in k^{*}$, whence $k[X]=k[F]$ by our hypothesis. Put $D_{j}:=\varphi\left(\partial_{j}\right)-b_{j}$ and write $\partial, D, b$ instead of $\partial_{1}, \ldots, \partial_{n}$ resp. $D_{1}, \ldots, D_{n}$ resp. $b_{1}, \ldots, b_{n}$. We have to prove that $\varphi$ is surjective, i.e. $\varphi(k[X, \partial])=k[X, \partial]$. So we must show that $k[F, D+b]=k[X, \partial]$. From $k[X]=k[F]$ we deduce $k[F, D+b]=k[X, D+b]=$ $k[X, D]$. Finally, $D_{j}=\sum a_{j k} \partial_{k}$ for all $i, j$. Since (11) implies $\left(a_{j k}\right) \in \mathrm{GL}_{n}(k[X])$ we derive that $\partial_{i} \in \sum_{k} k[X] D_{k}$ for all $i$. Hence $k[X, D]=k[X, \partial]$ which gives $k[F, D+b]=k[X, \partial]$ as desired.

\subsubsection{The Markus-Yamabe Conjecture}

Let $F: \mathbb{R}^{n} \rightarrow \mathbb{R}^{n}$ be a $\mathscr{C}^{1}$-vectorfield with $F(0)=0$. We say that $F$ satisfies the Markus-Yamabe condition if for all $x \in \mathbb{R}^{n}$ all eigenvalues of $J F(x)$ have a negative real part.

Furthermore we say that 0 is a global asymptotic restpoint of the autonomous system $\dot{y}=F(y)$ if each solution $\varphi(t, x)$ with $\varphi(0, x)=x$ tends to 0 if $t$ tends to infinity.

Conjecture 3.32 (Markus-Yamabe Conjecture (MYC(n)), [42])

Let $F: \mathbb{R}^{n} \rightarrow \mathbb{R}^{n}$ be a $\mathscr{C}^{1}$-vectorfield with $F(0)=0$ and satisfying the MarkusYamabe condition, then 0 is a global asymptotic restpoint of $\dot{y}=F(y)$.

If in the above conjecture we restrict to polynomial mappings the corresponding conjecture will be denoted by $\operatorname{PolMYC}(n)$. Before we describe the relation between these conjectures and the Jacobian Conjecture, let us first describe their status.

In 1988 PolMYC(2) was proved by Meisters and Olech in [44]. In 1993 MYC(2) was proved independently by Gutierrez in [29] and Feßler in [26]. For $n>3$ a counterexample to $M Y C(n)$ was given by Barabanov in [3]. (For a more extended version of this counterexample we refer to the paper [6].)

Recently in [9] the following counterexample to $\operatorname{PolMYC}$ was given for all $n \geq 3$ :

Counterexample 3.33 - Let

$$
F(X)=\left(-X_{1}+X_{3} d(X)^{2},-X_{2}-d(X)^{2},-X_{3}, \ldots,-X_{n}\right)
$$

where $d(X)=X_{1}+X_{3} X_{2}$. Then $\dot{x}=F(x)$ has a solution

$$
\begin{aligned}
X_{1}(t) & =18 e^{t} \\
X_{2}(t) & =-12 e^{2 t} \\
X_{3}(t) & =e^{-t} \\
& \vdots \\
X_{n}(t) & =e^{-t} .
\end{aligned}
$$

This clearly tends to infinity if $t$ tends to infinity. 
However the following weaker version of $\operatorname{PolMYC}(n)$ remains unsettled for all $n \geq 3$.

Conjecture 3.34 (Weak PolMYC(n)) - If $F: \mathbb{R}^{n} \rightarrow \mathbb{R}^{n}$ is a polynomial map satisfying the Markus-Yamabe condition, then $F$ is injective.

Finally we show:

Theorem 3.35 - If for all $n \geq 2$ the weak PolMYC(n) is true, then the Jacobian Conjecture is true.

Proof. Arguing as in chapter 1 (preceeding Yu's theorem) and using (1) it suffices to prove that all polynomial maps $F: \mathbb{R}^{n} \rightarrow \mathbb{R}^{n}$ of the form $-X+H$ with $J H$ nilpotent and $H$ homogeneous of degree three are injective. Now observe that the eigenvalues of $J F(x)=-I+J H(x)$ are all equal to -1 (since $J H(x)$ is nilpotent). Then apply weak $M Y C(n)$.

\section{References}

[1] S.S. Abhyankar, P. Eakin, and W. Heinzer, On the uniqueness of the coefficient ring in a polynomial ring, J. of Algebra 23 (1972), 310-342.

[2] S.S. Abhyankar and T.T. Moh, Embeddings of the line in the plane, J. Reine Angew. Math. 276 (1975), 148-166.

[3] N.E. Barabanov, On a problem of Kalman, Siberian Mathematical Journal 29 (1988), no. 3, 333-341.

[4] H. Bass, E. Connell, and D. Wright, The Jacobian Conjecture: Reduction of Degree and Formal Expansion of the Inverse, Bulletin of the American Mathematical Society 7 (1982), 287-330.

[5] A. Beauville, J.L. Colliot-Thélène, J.J. Sansuc, and P. Swinnerton-Dyer, Varietes stablement rationelles non rationelles, Annals of Mathematics 121 (1985), 283-318.

[6] J. Bernat and J. Llibre, Counterexamples to Kalman and Markus-Yamabe conjectures in dimension larger than 3, preprint 1994, to appear in Dynamics of Continuous, Discrete and Impulsive systems.

[7] A. Białynicki-Birula and M. Rosenlicht, Injective morphisms of real algebraic varieties, Proceedings of the American Mathematical Society 13 (1962), 200-203.

[8] C.C. Cheng and S.S.-S. Wang, Radial similarity of Newton polygons, In van den Essen [22], Proceedings of the conference 'Invertible Polynomial maps', pp. 157-168. 
[9] A. Cima, A.R.P. van den Essen, A. Gasull, E.-M.G.M. Hubbers, and F. Mañosas, A polynomial counterexample to the Markus-Yamabe Conjecture, Report 9551, University of Nijmegen, Toernooiveld, 6525 ED Nijmegen, The Netherlands, 1995.

[10] W. Danielewski, On the cancellation problem and automorphism group of affine algebraic varieties, Preprint, 1989.

[11] B. Deng, Automorphic conjugation, global attractor, and the Jacobian conjecture, preprint 1995.

[12] B. Deng, G.H. Meisters, and G. Zampieri, Conjugation for polynomial mappings, to appear in Z. Angew. Math. Phys. ZAMP.

[13] H.G.J. Derksen, More on the hypersurface $x+x^{2} y+z^{2}+t^{3}=0$ in $\mathbb{C}^{4}$, preprint 1995.

[14] The kernel of a derivation, J. of Pure and Applied Algebra 84 (1993), 13-16.

[15] J.K. Deveney and D.R. Finston, $G_{a}$-actions on $\mathbb{C}^{3}$ and $\mathbb{C}^{7}$, Comm. Alg 22 (1994), no. 15, 6295-6302.

[16] J. Dixmier, Sur les algèbres de Weyl, Bull. Soc. Math. France 96 (1968), 209-242.

[17] L.M. Drużkowski, An Effective Approach to Keller's Jacobian Conjecture, Math. Ann. 264 (1983), 303-313.

[18] The Jacobian Conjecture in case of rank or corank less than three, J. of Pure and Applied Algebra 85 (1993), 233-244.

[19] A.R.P. van den Essen, D-modules and the Jacobian Conjecture, Report 9108, University of Nijmegen, Toernooiveld, 6525 ED Nijmegen, The Netherlands, 1991.

[20] - Seven lectures on polynomial automorphisms, In Automorphisms of Affine Spaces [22], Proceedings of the conference 'Invertible Polynomial maps', pp. 3-40.

[21] $\longrightarrow$ A counterexample to a conjecture of Meisters, In Automorphisms of Affine Spaces [22], Proceedings of the conference 'Invertible Polynomial maps', pp. 231-234.

[22] (ed.), Automorphisms of Affine Spaces, Curaçao, Caribbean Mathematics Foundation, Kluwer Academic Publishers, July 4-8 1994, 1995, Proceedings of the conference 'Invertible Polynomial maps'.

[23] A.R.P. van den Essen and E.-M.G.M. Hubbers, Polynomial maps with strongly nilpotent Jacobian matrix and the Jacobian Conjecture, Report 
9444, University of Nijmegen, Toernooiveld, 6525 ED Nijmegen, The Netherlands, 1994, to appear in Linear Algebra and its Applications.

[24] A.R.P. van den Essen and E.-M.G.M. Hubbers, Chaotic Polynomial Automorphisms; counterexamples to several conjectures, Report 9549, University of Nijmegen, Toernooiveld, 6525 ED Nijmegen, The Netherlands, 1995.

[25] A.R.P. van den Essen and T. Janssen, Kernels of elementary derivations, Report 9548, University of Nijmegen, Toernooiveld, 6525 ED Nijmegen, The Netherlands, 1995.

[26] R. Feßler, A Solution to the Global Asymptotic Jacobian Conjecture and a Generalization, In workshop I-38050 POVO(TN), Italy, September 1993. To appear in Ann. Polon. Math.

[27] T. Fujita, On Zariski problem, Proc. Japan Acad. Ser. A, Math. Sci 55 (1979), 106-110.

[28] G. Gorni and G. Zampieri, On the existence of global analytic conjugations for polynomial mappings of Yagzhev type, preprint Univ. of Udine, Italy, July 1995.

[29] C. Gutierrez, A solution to the bidimensional Global Asymptotic Conjecture, In workshop I-38050 POVO(TN), Italy, September 1993.

[30] J. Gwoździewicz, Injectivity on one line, Bull. Soc. Sci. Łódź 7 (1993), 5960, Série: Recherches sur les déformationes XV.

[31] E.-M.G.M. Hubbers, The Jacobian Conjecture: Cubic Homogeneous Maps in Dimension Four, Master's thesis, University of Nijmegen, Toernooiveld, 6525 ED Nijmegen, The Netherlands, February 17 1994, directed by A.R.P. van den Essen.

[32] T. Kambayashi, Automorphism group of a polynomial ring and algebraic group actions on affine space, J. of Algebra 60 (1979), 439-451.

[33] M.-C. Kang, The biregular cancellation problem, J. of Pure and Applied Algebra 45 (1987), 241-253.

[34] A note on the birational cancellation problem, J. of Pure and Applied Algebra 77 (1992), 141-154.

[35] O. Keller, Ganze Cremona-Transformationen, Monatsh. Math. Phys. 47 (1939), 299-306.

[36] H. Kraft, Algebraic automorphisms of affine spaces, In Kraft et al. [38], proceedings of the conference 'Topological Methods in Algebraic Transformation Groups' held at Rutgers University, 4-8 April, 1988.

[37] Challenging problems on affine n-space, Sém. Bourbaki, 47ème année 802 (1994-1995). 
[38] H. Kraft, T. Petrie, and G.W. Schwarz (eds.), Topological methods in algebraic transformation groups, Progress in Math., vol. 80, Birkhäuser, BostonBasel-Berlin, 1989, proceedings of the conference 'Topological Methods in Algebraic Transformation Groups' held at Rutgers University, 4-8 April, 1988.

[39] J. Lang and S. Maslamani, Some results on the Jacobian Conjecture in higher dimension, J. of Pure and Applied Algebra 94 (1994), 327-330.

[40] A. Magnus, On polynomial solutions of a differential equation, Math. Scand. 3 (1955), 255-260.

[41] L. Makar-Limanov, On the hypersurface $x+x^{2} y+z^{2}+t^{3}$ in $\mathbb{C}^{4}$ or a $\mathbb{C}^{3}$-like threefold which is not $\mathbb{C}^{3}$, Preprint 1995.

[42] L. Markus and H. Yamabe, Global stability criteria for differential systems, Osaka Math. Journal 12 (1960), 305-317.

[43] G.H. Meisters, Polyomorphisms conjugate to Dilations, In van den Essen [22], Proceedings of the conference 'Invertible Polynomial maps', pp. 67-88.

[44] G.H. Meisters and C. Olech, Solution of the Global Asymptotic Stability Jacobian Conjecture for the Polynomial Case, Analyse Mathématique et Applications, Gauthier-Villars, Paris, 1988, pp. 373-381.

[45] M. Miyanishi and T. Sugie, Affine surfaces containing cylinderlike open sets, J. Math Kyoto Univ. 20 (1980), 11-42.

[46] M. Nagata, a revised version of both [48] and [49].

[47] - On the fourteenth problem of Hilbert, Proceedings Int. Congress Math. 1958 (New York), Cambridge University, Cambridge Univ. Press, 1966, pp. 459-462.

[48] Two dimensional jacobian conjecture, Proceedings of the $3^{\text {rd }}$ KIT Mathematics Workshop (Taejŏn) (M.H. Kim and K.H. Ko, eds.), Korean Institute of Technology, 1988, MR 90k:14010a, Zbl.712.13003, pp. $77-98$.

Some remarks on the two-dimensional Jacobian Conjecture, Chin. J. Math. 17 (1989), 1-7.

[50] M. Nagata and A. Nowicki, Rings of constants for $k$-derivations in $k\left[x_{1}, \ldots, x_{n}\right]$, J. Math. Kyoto Univ. 28 (1988), no. 1, 111-118.

[51] P. Nousiainen and M. Sweedler, Automorphisms of polynomial and powerseries rings, J. of Pure and Applied Algebra 29 (1983), 93-97.

[52] A. Nowicki, Commutative basis of derivations in polynomial and powerseries rings, J. of Pure and Applied Algebra 40 (1986), 279-283. 
[53] A. Nowicki and J.-M. Strelcyn, Generators of rings of constants for some diagonal derivations in polynomial rings, to appear in J. of Pure and Applied Algebra.

[54] P. Roberts, An infinitely generated symbolic blow-up in a power series ring and a new counterexample to hilbert's fourteenth problem, J. of Algebra 132 (1990), 461-473.

[55] L. Rudolph, Embeddings of the line in the plane, J. Reine Angew. Math. 337 (1982), 113-118.

[56] G. Schwarz, Exotic algebraic group actions, C.R. Acad. Sci. Paris 309 (1989), 89-94.

[57] T. Sugie, Algebraic characterization of the affine plane and the affine threespace, In Kraft et al. [38], proceedings of the conference 'Topological Methods in Algebraic Transformation Groups' held at Rutgers University, 4-8 April, 1988, pp. 177-190.

[58] S.S.-S. Wang, A Jacobian criterion for separability, J. of Algebra 65 (1980), 453-494, MR 83e:14010, Zbl.471.13005.

[59] D. Wright, On the Jacobian Conjecture, Illinois J. of Math. 15 (1981), no. 3, $423-440$.

[60] - The Jacobian Conjecture: linear triangularization for cubics in dimension three, Linear and Multilinear Algebra 34 (1993), 85-97.

[61] A.V. Yagzhev, On Keller's problem, Siberian Math. J. 21 (1980), 747-754.

[62] J.-T. Yu, On the Jacobian Conjecture: reduction of coefficients, J. of Algebra 171 (1995), 515-523.

[63] A. Zaks, Dedekind subrings of $k\left[x_{1}, \ldots, x_{n}\right]$ are rings of polynomials, Israel J. of Mathematics 9 (1971), 285-289.

[64] G. Zariski, Interprétations algébrico-géométriques du quatorzième problème de Hilbert, Bull. Sci. Math. 78 (1954), 155-168. 